
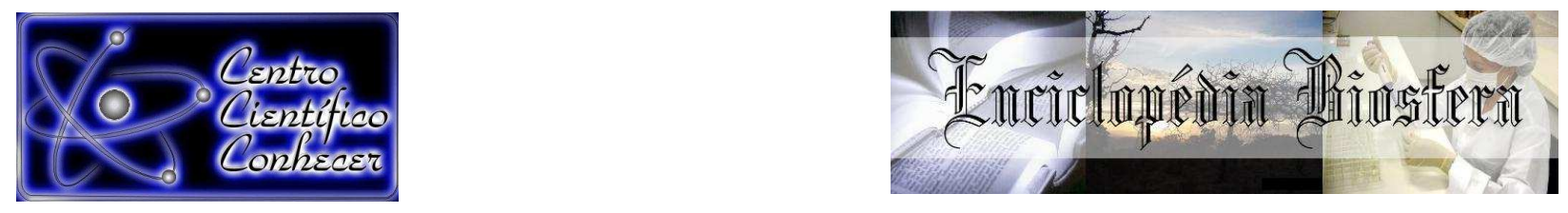

\title{
ANÁLISE DA PRODUÇÃO CIENTÍFICA SOBRE A TECNOLOGIA ASSISTIVA NO BRASIL
}

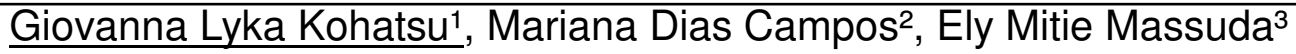

1Mestranda do Programa de Pós Graduação em Promoção da Saúde (PPGPS) do Centro Universitário de Maringá (gii.kohatsu@hotmail.com). Maringá, Paraná, Brasil.

${ }^{2}$ Acadêmica do Curso de Medicina, Centro Universitário de Maringá -

UNICESUMAR, Maringá, PR. Bolsista PIBIC/Fundação Araucária.

${ }^{3}$ Docente Pós Graduação em Promoção da Saúde (PPGPS) do Centro Universitário de Maringá; Pesquisadora do Instituto Cesumar de Ciência, Tecnologia e Inovação

Recebido em: 02/10/2017 - Aprovado em: 21/11/2017 - Publicado em: 05/12/2017 DOI: 10.18677/EnciBio 2017B92

\begin{abstract}
A tecnologia assistiva é um recurso indispensável para pessoas portadoras de deficiência, visto o benefício auferido na qualidade de vida e inclusão na sociedade desses indivíduos. Por meio da análise cienciométrica, o objetivo do presente estudo foi de avaliar a produção científica sobre a temática nos últimos oito anos, nas bases Scielo, PubMed e na Biblioteca Virtual de Saúde (BVS), nas línguas portuguesa e inglesa. Foram selecionados 33 artigos, dos quais a maior concentração ocorreu em 2017. O estado de São Paulo destacou-se pela maior quantidade de publicações e quanto ao periódico, evidenciou-se a Revista Brasileira de Educação Especial. Em termos de classificação Qualis/Capes, prevaleceu o estrato A1 e B1. Quanto a classificação por tipo de deficiência e por público alvo, houve dificuldade em definilas por apresentaram maior prevalência nos itens "outros" e "não consta". Concluiuse que a tecnologia assistiva é ainda pouco explorada no âmbito da pesquisa, e ainda assim, observando-se a dificuldade quanto às classificações por tipo de deficiência e público alvo. São relativamente escassos os artigos publicados sobre a temática.
\end{abstract}

RESUMO

PALAVRAS-CHAVE: pessoa com deficiência, publicações, tecnologia assistiva.

\section{ANALYSIS OF SCIENTIFIC PRODUCTION ON ASSISTIVE TECHNOLOGY IN BRAZIL}

\begin{abstract}
Assistive technology is an indispensable resource for people with disabilities, given the benefit they have on the quality of life and inclusion in society of these individuals. The objective of the present study was to evaluate the scientific production on the subject in the last eight years, in the bases Scielo, PubMed and in the Virtual Health Library (VHL), in the Portuguese and English languages. A total of 33 articles were selected, of which the highest concentration occurred in 2017 . The state of São Paulo stood out due to the greater number of publications and as for the periodical, it was evidenced the Brazilian Journal of Special Education. In terms of Qualis / Capes classification, stratum A1 and B1 prevailed. Regarding the
\end{abstract}


classification by type of disability and by the target public, it was difficult to define them because they presented higher prevalence in the "other" and "not in" items. It was concluded that the assistive technology is still little explored in the scope of the research, and yet, noting the difficulty regarding classifications by type of disability and target audience. The published articles about the subject are relatively scarce.

KEYWORDS: assistive technology, people with disabilities, publications.

\section{INTRODUÇÃO}

De acordo com definição estabelecida pelo Comitê de Ajudas Técnicas (CAT), tecnologia assistiva (TA) trata-se de:

(...) área do conhecimento, de característica interdisciplinar, que engloba produtos, recursos, metodologias, estratégias, práticas e serviços que objetivam promover a funcionalidade, relacionada à atividade e participação, de pessoas com deficiência, incapacidades ou mobilidade reduzida, visando sua autonomia, independência, qualidade de vida e inclusão social. (BRASIL, 2009, p. 9).

Admite-se que sua existência, como garantia de autonomia e independência para os indivíduos, vem desde os primórdios da humanidade com a utilização, por exemplo, de pedaços de madeira como órtese, pelos homens primitivos. Devido à significativa parcela da população que é portadora de algum tipo de deficiência, temse a necessidade de desenvolvimento cada vez maior desta área, visando melhor eficácia de recursos e acessibilidade aos indivíduos, garantindo inclusão social e qualidade de vida (RODRIGUES; ALVES, 2013).

O CAT foi instituído em 16 de novembro de 2006, pela Portaria $\mathrm{n}^{0}$ 142, estabelecida pelo Decreto n 5.296/2004, no domínio da Secretaria Especial dos Direitos Humanos, regulamentado pelo Poder Executivo e supervisionado pela Coordenadoria Nacional para Integração da Pessoa Portadora de Deficiência CORDE, sendo parte do Programa Nacional de Acessibilidade (BRASIL, 2009). Tem como objetivos principais:

(...) apresentar propostas de políticas governamentais e parcerias entre a sociedade civil e órgãos públicos referentes à área de tecnologia assistiva; estruturar as diretrizes da área de conhecimento; realizar levantamento dos recursos humanos que atualmente trabalham com o tema; detectar os centros regionais de referência, objetivando a formação de rede nacional integrada; estimular nas esferas federal, estadual, municipal, a criação de centros de referência; propor a criação de cursos na área de tecnologia assistiva, bem como o desenvolvimento de outras ações com o objetivo de formar recursos humanos qualificados e propor a elaboração de estudos e pesquisas, relacionados com o tema da tecnologia assistiva (BERSCH, 2013, p. 3).

Ademais, teve a execução integral de determinações garantidas por meio do Decreto no 6949, assinado em agosto de 2009, que aprovou a Convenção sobre os Direitos das Pessoas com Deficiência, e que destaca a TA como uma das formas de integração das pessoas com deficiência (CRUZ; EMMEL, 2013).

Além das legislações supracitadas, no Brasil, algumas outras políticas públicas estão contribuindo para a ampliação do uso de TA pelas pessoas com deficiência. Dentre estas, destaca-se a Política Nacional de Educação Especial, que visa a inclusão dos indivíduos com deficiência, nas diferentes faixas etárias, em escolas regulares, necessitando para isso, o uso de TA visando a facilitação do 
processo de aprendizagem dos mesmos. Outra política que merece destaque é o Plano Nacional dos Direitos da Pessoa com Deficiência - Plano Viver sem Limites, que facilita o acesso aos recursos de TA via criação de linha de crédito para aquisição dos mesmos, e pelo investimento em pesquisa e desenvolvimentos de novos recursos (RODRIGUES; ALVES, 2013).

A respeito da classificação para os recursos que compõe a TA, três referências importantes são consideradas. Uma trata-se de um modelo europeu, o HEART - Horizontal European Activities in Rehabilitation Technology e que toma como base os conhecimentos envolvidos na utilização, dividindo os recursos em três grandes áreas: componentes técnicos, humanos e socioeconômicos. A ISO 9999 International Organization for Standardization, uma federação mundial com influência sobre diversos países, estipula outra classificação, a qual é utilizada na forma de bases de dados e catálogos, e baseia-se principalmente nos recursos e nas respectivas funções para classificá-los, organizando-os em classes, nas quais se enquadram os produtos, que variam desde exclusivos a pessoas com deficiência a produtos com versões especiais para as mesmas. Uma terceira que merece destaque é a Classificação Nacional de Tecnologia Assistiva do Departamento de Educação dos Estados Unidos que se fundamenta nas áreas de aplicação dos recursos para classificá-los e subdividindo-os em 10 itens de componentes, além de atuar no desenvolvimento, customização de recursos e apoio aos usuários (BRASIL, 2009).

Segundo levantamento realizado pela Organização Mundial de Saúde e divulgado por meio do Relatório Mundial sobre Deficiência (World Report on Disability), aproximadamente $15 \%$ da população mundial possui algum tipo de deficiência em 2011, e destas, $20 \%$ enfrentam grandes dificuldades no cotidiano (CRUZ; EMMEL, 2013).

No Brasil, de acordo com o levantamento realizado pelo Instituto Brasileiro de Geografia e Estatística (IBGE) e divulgado pelo Censo 2010, 23,9\% da população possui algum tipo de deficiência, dado que enfatiza o padrão crescente da incidência e prevalência da deficiência no Brasil. O Censo de 2001 revelou que $14,5 \%$ da população do país era composta por pessoas com deficiência. Associada à magnitude desses dados, percebe-se uma alteração na concepção da sociedade em geral com relação à necessidade de inclusão social desta parcela da população por meio da mobilização de ações que visem garantir a participação de todos no meio social, independente das características físicas, ressaltando ainda mais a necessidade de recursos de TA para facilitar tal processo (RODRIGUES; ALVES, 2013).

Um problema, porém, que ainda deve ser resolvido com relação a estes recursos, segundo Varela e Oliver (2013), é o elevado custo no mercado nacional, uma vez que há pouca disponibilidade do material produzido pelas universidades brasileiras, restando como opção aos compradores a importação que acaba acarretando aumento no valor total do produto, uma vez que a maior parte destes dispositivos não possuem isenção tributária. Soma-se a este fator, a prevalência da baixa renda entre as famílias das pessoas com deficiência, tendo como consequência o acesso restrito aos bens e serviços, pois os gastos decorrentes dos cuidados com estes indivíduos acabam assumindo parcela significativa de seus proventos (SOUZA; CARNEIRO, 2007).

Em análise realizada por Garcia e Galvão Filho (2012), sobre as inovações na área de TA no Brasil, algumas conclusões refletem a maior necessidade de intervenções e ampliações sobre o tema no país: por exemplo, o fato da maior parte 
dos projetos serem concentrados nos estados de Rio de Janeiro, São Paulo e Rio Grande do Sul, sendo escassa a ocorrência nos estados do Nordeste, região, segundo o Censo de 2010, onde se concentra a maior parte das pessoas com deficiência no Brasil (IBGE, 2012). Ademais, demonstra a existência de discrepância entre a porcentagem de pesquisas sobre TA desenvolvidas no Brasil, aproximadamente $52 \%$ dos projetos, e o desenvolvimento de produtos - que ocorreram em $23 \%$ dos projetos, evidenciando a necessidade de maior produção dos recursos que estão sendo pesquisados (GARCIA; GALVÃO FILHO, 2012).

Com base nessas considerações que evidenciam a carência de estudos sobre a temática no Brasil e a necessidade do desenvolvimento de mais pesquisas que valorizem a importância na vida cotidiana das pessoas com deficiência, o presente trabalho apresenta um estudo cenciométrico a fim de avaliar a produção científica sobre a TA no Brasil.

\section{MATERIAL E MÉTODOS}

Realizou-se um estudo cenciométrico sobre a TA no Brasil, por meio das bases de dados indexadas no United States National Library of Medicine (PubMed), que possui como acesso o site http://www.ncbi.nlm.nhi.gov/pubmed, no Scientific Eletronic Library Online (SciELO), disponível no site http://www.scielo.org/php/index.php, e na Biblioteca Virtual em Saúde (BVS) acessada pelo site http://brasil.bvs.br/. Não houve a aplicação de filtros devido à escassez de artigos publicados acerca do tema. A pesquisa foi feita nos meses de agosto e setembro de 2017 utilizando-se as terminologias "Tecnologia assistiva" e "Brasil", e em inglês "Assistive Technology" e "Brazil".

Os artigos foram selecionados a partir da análise do título, resumo e palavraschave, sendo excluídos quaisquer artigos que não abordassem a terminologia "Tecnologia Assistiva" nesses requisitos e artigos indisponíveis online. Depois da realização da leitura dos resumos, estes foram classificados pelo ano de publicação, local de origem das publicações (baseado no primeiro autor), tipo de deficiência abordada, público-alvo e o Qualis/Capes correspondente às revistas, sendo selecionados todas as áreas de avaliação, por meio de planilhas no programa Microsoft Excel 2010.

\section{RESULTADOS E DISCUSSÃO}

A pesquisa apresentou algumas particularidades acerca da forma pela qual a TA está inserida no campo da pesquisa, como será visto adiante. No processo inicial foram encontradas 124 publicações científicas, sendo 34 da base de dados BVS, 79 da PubMed e 11 do Scielo. Após a leitura e avaliação dos títulos, resumos e palavras-chave e instituído os critérios de exclusão, restaram 33 artigos.

A partir da Figura 1 pode-se observar que no estado de São Paulo a prevalência da origem dos artigos é maior (14), seguido do Ceará (5), Bahia (3), Goias (2), Paraná (2), entre outros. Confirma-se como São Paulo o estado que mais produz artigos sobre a TA (GARCIA, GALVÃO FILHO, 2012). 


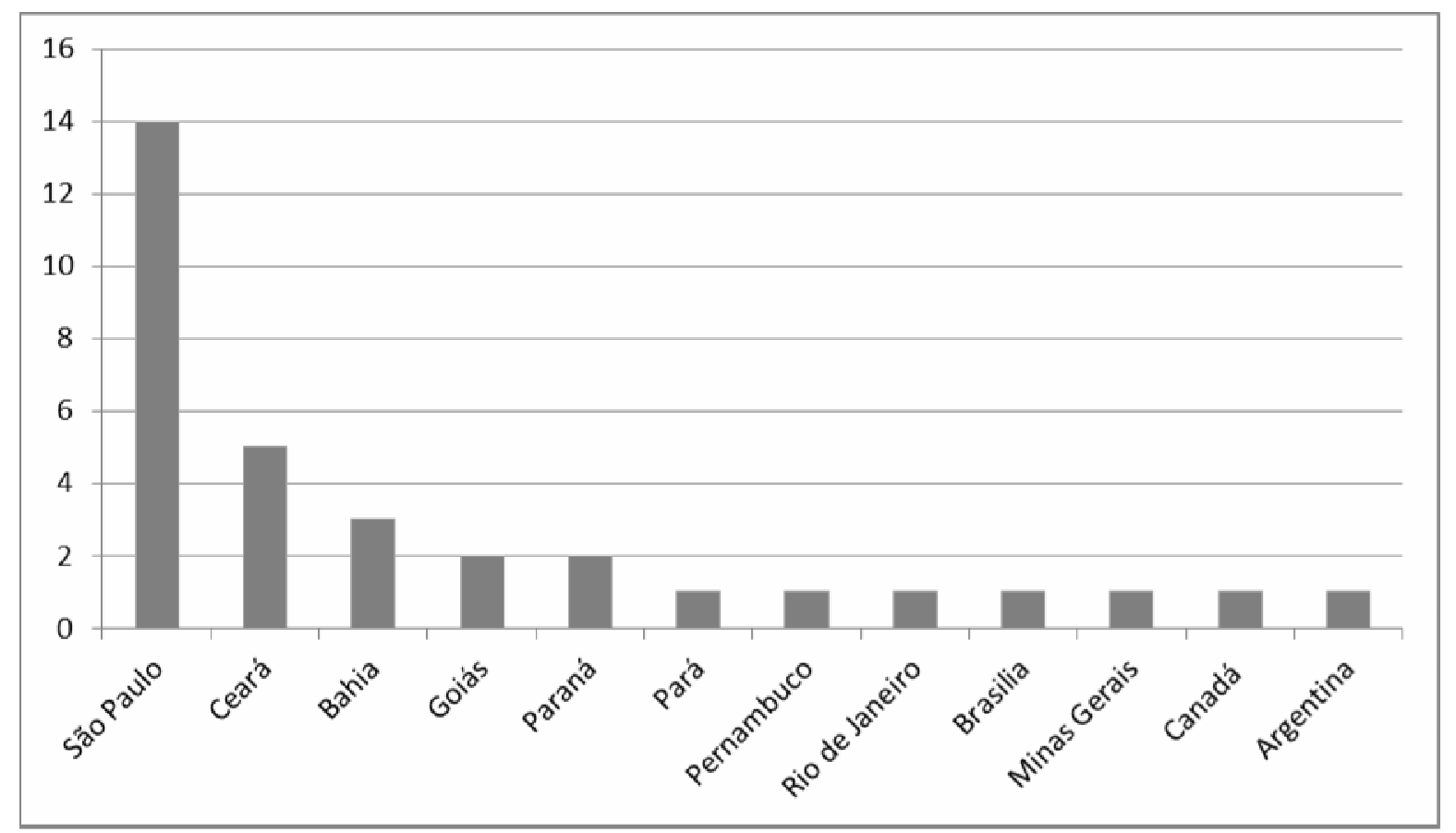

FIGURA 1- Locais de origem das publicações

$\mathrm{Na}$ figura 2, observa-se que entre o ano de 2009 e 2010 houve leve queda nas publicações sobre TA, entre 2011 a 2015 ocorreu um aumento significativo, declínio em 2016, apresentando-se tendência de expansão em 2017. Há que se ressaltar que a abordagem desse tema é muito recente, tendo em vista que somente em 2006 a Secretaria Especial dos Direitos Humanos estabeleceu um Comitê de Ajudas Técnicas (CAT) como diversos objetivos para inserir a tecnologia assistiva na sociedade, dentre estas a área da pesquisa (BERSCH, 2013).

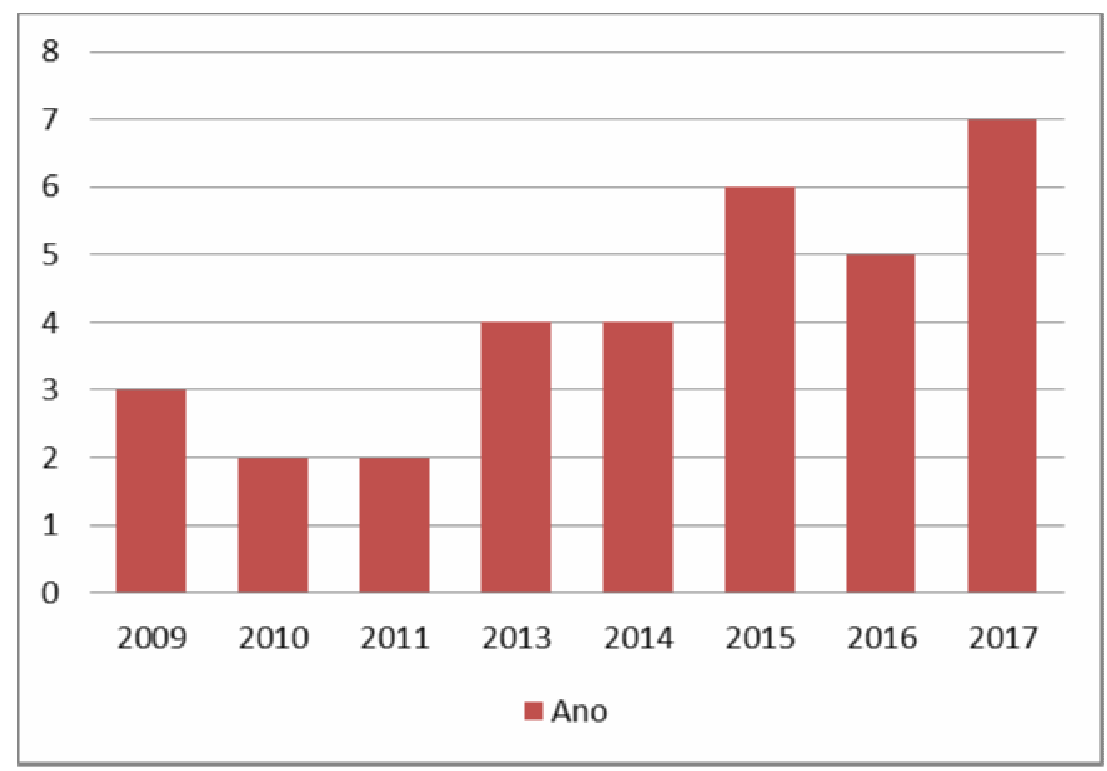

FIGURA 2 - Quantidade de artigos publicados do ano de 2009 a 2017

$\mathrm{Na}$ realização da leitura dos artigos houve dificuldade em definir o tipo de deficiência e a população alvo abordada pelos artigos, impossibilitando determinar concretamente estes resultados. Conforme se observa na Figuras 3, na definição dos tipos de deficiências abordadas no artigos levantados, constatou-se a 
prevalência de "outros" e "não consta" não se permitindo inferir a deficiência predominante. No entanto, entre aquelas classificadas, artigos que tratam de deficiência visual são as mais representativas.

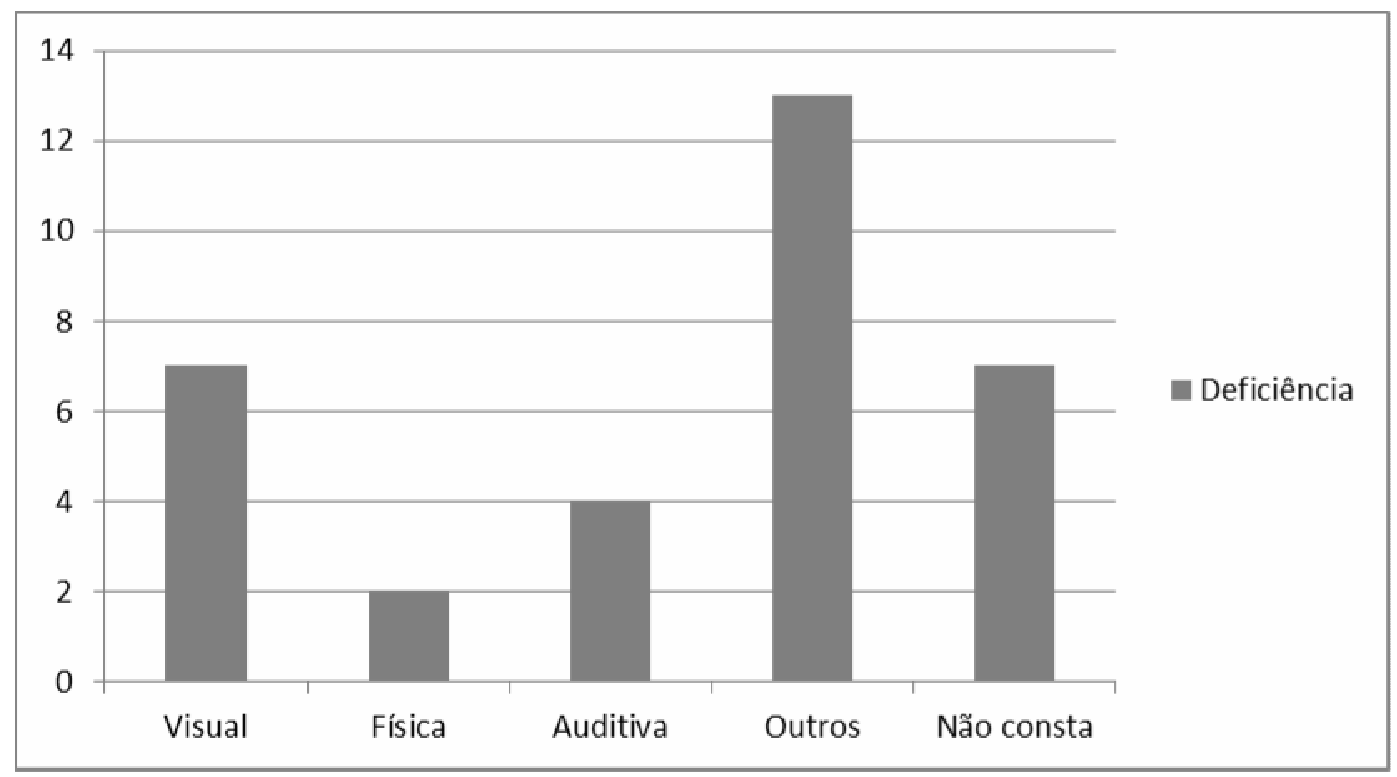

FIGURA 3 - Tipos de deficiência tratados nos artigos

Conforme a Figura 4, a mesma situação se repete quanto a indefinição do público alvo dado o elevado número tomado pelas classificações "outros" e "não consta". Da mesma forma, dentre aqueles identificados, professores constituem o público alvo mais discutido nos artigos.

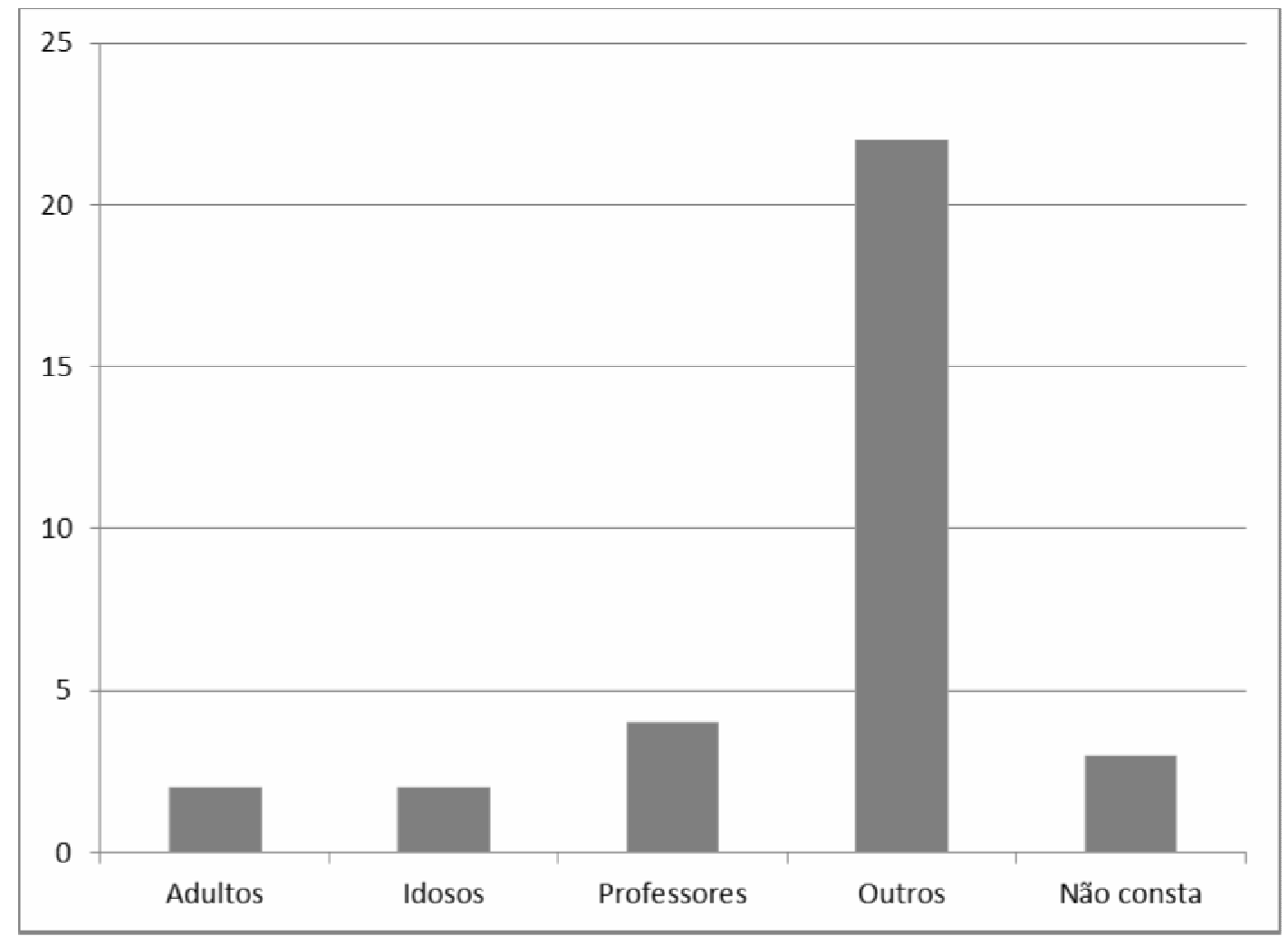

FIGURA 4 - Público alvo abordado nos artigos indexados 
No que se refere aos periódicos, a Revista Brasileira de Educação Especial correspondeu ao maior número de publicações acerca do tema (5), seguida da Revista da Escola de Enfermagem da USP (4), Revista Brasileira de Reumatologia (3), Disability and Rehab: Assistive Technology (3), entre outros (Tabela 1).

TABELA 1 - Publicações em Revistas sobre TA

\begin{tabular}{lc}
\hline REVISTA & No PUBLICAÇÕES \\
\hline Revista Brasileira de Educação Especial & 5 \\
\hline Revista da Escola de Enfermagem da USP & 4 \\
\hline Revista Brasileira de Reumatologia & 3 \\
\hline Disability and Rehab.: Assistive Technology & 3 \\
\hline CODAS & 2 \\
\hline Revista Panamericana de Salud Publica & 2 \\
\hline Archives of Physical Medicine and Rehab & 1 \\
\hline BMJ & 1 \\
\hline Revista da Sociedade Brasileira de Medicina & 1 \\
Tropical & 1 \\
\hline Ciência e Saúde Coletiva & 1 \\
\hline Clinical Neurology and Neurosurgery & 1 \\
\hline Journal of Clinical and Diagnostic Research & 1 \\
\hline Neurosurgical Review & 1 \\
\hline PLOS & 1 \\
\hline PLOS ONE & 1 \\
\hline Revista Brasileira de Enfermagem & 1 \\
\hline Revista Eletrônica de Administração (REad) & 1 \\
\hline Revista de Terapia Ocupacional da USP & 1 \\
\hline Special Care in Dentistry & 1 \\
\hline The Scientific World Journal & 33 \\
\hline TOTAL &
\end{tabular}

O quadro 1 mostra a classificação, quadriênio 2013-2016, dos periódicos que publicaram artigos acerca das tecnologias assistivas e as respectivas áreas de avaliação que apresentaram maior estrato Qualis. Observa-se que dos 20 periódicos, sete (35\%) possuem Qualis A1; cinco (25\%), Qualis A2; sete $(35 \%)$, Qualis B1; 1 (5\%), Qualis B4. Portanto, a 95\% estão elencadas em estrato superior. A Revista Brasileira de Educação Especial que possui maior número de publicações sobre TA está classificada como A1 na área de Ensino.

QUADRO 1- Classificação do Qualis/Capes em todas as áreas de avaliação

\begin{tabular}{|c|c|c|}
\hline & & Qualis de maior estrato \\
\hline Revista & Qualis & Área de Avaliaccão \\
\hline Ciência e Saúde coletiva & A1 & Ensino; Serviço Social e Sociologia \\
\hline $\begin{array}{l}\text { Revista Brasileira de } \\
\text { Reumatologia }\end{array}$ & $\mathrm{A} 1$ & Arquitetura, Urbanismo e Design \\
\hline $\begin{array}{c}\text { Revista Brasileira de Educação } \\
\text { Especial }\end{array}$ & A1 & Ensino \\
\hline
\end{tabular}




\begin{tabular}{|c|c|c|}
\hline CODAS & A1 & Linguística e Literatura \\
\hline PLOS ONE & A1 & $\begin{array}{l}\text { Biodiversidade; Biotecnologia; Ciência } \\
\text { de Alimentos; Ciência Política e } \\
\text { Relações Internacionais; Ciências } \\
\text { Agrárias I; Ciências Ambientais; } \\
\text { Educação Física; Engenharias III; } \\
\text { Geografia; Interdisciplinar; Medicina } \\
\text { Veterinária; Odontologia; Psicologia; } \\
\text { Saúde Coletiva; Sociologia; Zootecnia/ } \\
\text { Recursos Pesqueiros. }\end{array}$ \\
\hline $\begin{array}{l}\text { Archives of Physical Medicine } \\
\text { and Rehab }\end{array}$ & $\mathrm{A} 1$ & Educação Física; Interdisciplinar \\
\hline PLOS & A1 & $\begin{array}{l}\text { Biotecnologia; Ciências Agrárias I; } \\
\text { Ciências Ambientais; Enfermagem; } \\
\text { Interdisciplinar; Medicina Veterinária; } \\
\text { Odontologia; Planejamento Urbano e } \\
\text { Regional/ Demografia; Saúde Coletiva; } \\
\text { Zootecnia/ Recursos Pesqueiros }\end{array}$ \\
\hline $\begin{array}{l}\text { Revista Panamericana de Salud } \\
\text { Publica }\end{array}$ & A2 & $\begin{array}{c}\text { Administração Pública e de Empresas, } \\
\text { Ciências Contábeis e Turismo; } \\
\text { Educação; Planejamento Urbano e } \\
\text { Regional/ Demografia; Psicologia; } \\
\text { Saúde Coletiva }\end{array}$ \\
\hline $\begin{array}{c}\text { Revista da Sociedade Brasileira } \\
\text { de Medicina Tropical }\end{array}$ & A2 & Ciências Ambientais; Geografia \\
\hline $\begin{array}{l}\text { Revista da Escola de } \\
\text { Enfermagem da USP }\end{array}$ & A2 & Educação; Enfermagem; Psicologia \\
\hline The Scientific World Journal & A2 & $\begin{array}{l}\text { Administração Pública e de Empresas; } \\
\text { Ciências Contáveis e Turismo }\end{array}$ \\
\hline $\begin{array}{l}\text { Revista Brasileira de } \\
\text { Enfermagem }\end{array}$ & A2 & Enfermagem; Psicologia \\
\hline $\begin{array}{c}\text { Revista de Terapia Ocupacional } \\
\text { da USP }\end{array}$ & B1 & Educação Física \\
\hline Neurosurgical Review & B1 & Medicina II; Medicina III \\
\hline Special Care in Dentistry & B1 & $\begin{array}{l}\text { Ciências Ambientais; Enfermagem; } \\
\text { Odontologia }\end{array}$ \\
\hline $\begin{array}{l}\text { Journal of Clinical and } \\
\text { Diagnostic Research }\end{array}$ & B1 & Ciências Ambientais \\
\hline $\begin{array}{l}\text { Disability and Rehab.: Assistive } \\
\text { Techonology }\end{array}$ & B1 & Educação Física; Enfermagem \\
\hline $\begin{array}{l}\text { Revista Eletrônica de } \\
\text { Administração (REad) }\end{array}$ & B1 & $\begin{array}{c}\text { Administração Pública e de Empresas, } \\
\text { Ciências Contábeis e Turismo; } \\
\text { Ciências Ambientais; Economia; } \\
\text { Planejamento Urbano e Regional/ } \\
\text { Demografia; Psicologia }\end{array}$ \\
\hline $\begin{array}{l}\text { Clinical Neurology and } \\
\text { Neurosurgery }\end{array}$ & B1 & Interdisciplinar \\
\hline BMJ & B4 & Medicina III \\
\hline
\end{tabular}


As temáticas abordadas pelos artigos selecionados apresentaram perspectivas distintas. A atuação dos profissionais da saúde na educação, a utilização de tecnologias assistivas para a melhora da qualidade de vida e inclusão social das pessoas portadoras de deficiência foram os focos de pesquisas conduzidas por Alves et al. (2009), Hohmamm e Cassapian (2017) e Borges e Tartuci, (2017).

A utilização de software para as pessoas portadoras de deficiência foram referidas em publicações relativas à tecnologia assistiva: utilização de ensino de palavras em libras por meio da tecnologia de realidade aumentada (CARVALHO, MANZINI, 2017); desenvolvimento de novos tipos de tecnologia assistivas (MEDOLA, et al, 2011; PEREZ, et al, 2013); percepção e o empoderamento de pessoas deficiências visuais para a amamentação e utilização de preservativos (OLIVEIRA et al., 2009); tecnologia assistiva para o deficiente visual na utilização do preservativo masculino (BARBOSA, et al, 2013); tecnologia assistiva para mulheres com deficiência visual acerca do preservativo feminino (CAVALCANTE, et al., 2015).

Outros estudos focaram algum tipo de doença genética ou adquirida com potencial de levar o portador a alguma deficiência como a distrofia muscular de Duchenne (DMD), lepra, distonia muscular idiopática generalizada, acidente vascular cerebral crônico, glioma. Nessa perspectiva, as pesquisas foram orientadas para o uso da tecnologia assistiva no auxílio ao cotidiano das pessoas, sua inclusão social ou como instrumento em cirurgias dessas patologias (BARBOSA, et al., 2014; GUIMARÃES et al., 2016; BARBOSA, et al., 2016; MUNIZ et al., 2016; MAIA et al., 2016; ALVAREZ et al., 2017; GAMA et al., 2017).

Alguns dos artigos selecionados apresentaram enfoques específicos como a redução de biofilme das próteses dentárias de idosos a partir de uma tecnologia assistiva (KAMMERS, et al, 2015); a correlação da tecnologia assistiva com o poder aquisitivo, a independência da vida diária e papéis ocupacionais em pessoas portadoras de deficiência física (CRUZ; EMMEL, 2013); a inserção no mercado de trabalho bem como o treinamento, desenvolvimento e a adequação das condições de trabalho para pessoas com deficiência (MAIA; FREITAS, 2015); epidemiologia de doenças genéticas e adquiridas que causam deficiência física bem como estimativa de custos para a obtenção das tecnologias assistivas e de serviços como fisioterapia (SANTOS et al., 2014); acesso às tecnologias assistivas para populações com idade acima de 60 anos em países com renda média e baixa (MARASINGHE et al., 2015).

Entre as pesquisas publicadas existem aquelas voltadas para a validação de instrumentos de avaliação de uso de tecnologias assistivas, tais como: tradução para o português e validação de instrumento de avaliação da satisfação de usuários de tecnologia assistiva - QUEST 2.0 (CARVALHO et al., 2014); adaptação de instrumento de avaliação auditiva para crianças (JACOB et al., 2010); adaptação transcultural do dispositivo de tecnologia assistiva para uso no Brasil (ALVES et al., 2016); tradução e adaptação cultural para a língua portuguesa do instrumento TELEGRAM e avaliação da aplicabilidade em adultos com deficiência auditiva usuários aparelho de Amplificação Sonora Individual, uma da tecnologia assistiva (JACOB at al., 2017).

\section{CONCLUSÃO}

A ênfase sobre papel desempenhado pelas tecnologias assistivas na inserção social e na qualidade de vida das pessoas com deficiência ainda é recente, de forma que ainda são relativamente escassos os artigos divulgados sobre a temática. As publicações não permitiram inferir os tipos de deficiência tratados nos artigos ou o 
público alvo abordado. Contudo, as publicações estão inseridas em periódicos em estratos superiores, na maioria.

\section{REFERÊNCIAS}

ALVAREZ, M. P. B.; SILVA, T. D.; FAVERO, F. M.; VALENTI, V. E.; RAIMUNDO, R. D.; et al.; Autonomic Modulation in Duchenne Muscular Dystrophy during a Computer Task: A Prospective Control Trial. PLOS ONE, 2017. Disponível em: $<\mathrm{http}$ ://journals.plos.org/plosone/article?id=10.1371/journal.pone.0169633>. DOl: 10.1371/journal.pone.0169633

ALVES, A. C. J.; MATSUKURA, T. S.; SCHERER, M. J. Cross-cultural adaptation of the assistive technology device - Presdisposition assessment (ATD PA) for use in Brazil (ATD PA Br). Disability and Rehabilitation: Assistive Technology. v. 12, n. 2, p. 160-164. 2016. Disponível em: <http://dx.doi.org/10.1080/17483107.2016.1233294>. DOI: 10.1080/17483107.2016.1233294

ALVES, C. C. F.; MONTEIRO, G. B. M.; RABELLO, S.; GASPARETTO, M. E. R. F.; CARVALHO, K, M. Assistive technology applied to education of students with visual impairment. Revista Panamericana de Salud Publica. v. 26, n. 2, p. 148-152. 2009. Disponível em: < http://dx.doi.org/10.1590/S1020-49892009000800007>. DOI: 10.1590/S1020-49892009000800007

BARBOSA, B. J. A. P.; MARIANO, E. D.; BATISTA, C. M.; MARIE, S. K. N.; TEIXEIRA, M. J.; PEREIRA, C. U.; et al.,; Intraoperative assistive technologies and extent of resection in glioma surgery: a systematic review of prospective controlled studies. Neurosurgical Review. n. 38, p. 217-227. 2015. Disponível em: <https://doi.org/10.1007/s10143-014-0592-0>. DOI: 10.1007/s10143-014-0592-0

BARBOSA, B. J. A. P.; DIMOSTHENI, A.; TEIXEIRA, M. J.; TATAGIBA, M.; LEPSKI, G. Insular gliomas and the role of intraoperative assistive technologies: Results from a volumetry0based retrospective cohort. Clinical Neurology and Neurosurgery. v. 149, p. 104-110. 2016. Disponível em: <https://doi.org/10.1016/j.clineuro.2016.08.001>. DOI: 10.1016/j.clineuro.2016.08.001

BARBOSA, G. O. L.; WANDERLLEY, L. D.; REBOUÇAS, C. B. A.; OLIVEIRA, P. M. P.; PAGLIUCA, L. M. F. Desenvolvimento de tecnologia assistiva para o deficiente visual: utilização do preservativo masculino. Revista da Escola de Enfermagem da

USP. v. 47, n. 5, p. 1165-1171. 2013. Disponível em: <http://dx.doi.org/10.1590/reeusp.v47i5.78075>. DOI: 10.1590/reeusp.v47i5.78075

BERSCH, R. Introdução à Tecnologia Assistiva. Porto Alegre, RS: [s.n.], 2013. Disponível em: < http://www.assistiva.com.br/Introducao_Tecnologia_Assistiva.pdf>. Acesso em: 20 set. 2017.

BRASIL. Subsecretaria Nacional de Promoção dos Direitos da Pessoa com Deficiência. Comitê de Ajudas Técnicas. Tecnologia Assistiva. Brasília: CORDE, 2009.

Disponível

em: 
$<$ http://www.pessoacomdeficiencia.gov.br/app/sites/default/files/publicacoes/livrotecnologia-assistiva.pdf>. Acesso em: 20 set. 2017.

BORGES, W. F.; TARTUCI, D. Tecnologia Assistiva: Concepções de Professores e as Problematizações Geradas pela Impressão Conceitual. Revista Brasileira de Educação Especial. v. 23, n. 1, p. 81-96. 2017. Disponível em: <http://dx.doi.org/10.1590/s1413-65382317000100007>. DOI: 10.1590/s141365382317000100007

CARVALHO, K. E. C.; JUNIOR, M. B. G.; SÁ, K. N. Tradução e validação do Quebec User Evaluation of Satisfaction with Assistive Technology (QUEST 2.0) para o idioma português do Brasil. Revista Brasileira de Reumatologia. v. 54, n. 4, p. 260-267. 2014. Disponível em: <http://dx.doi.org/10.1016/j.rbr.2014.04.003>. DOI: 10.1016/j.rbr.2014.04.003

CARVALHO, D.; MANZINI, E. J. Aplicação de um Programa de Ensino de Palavras em Libras utilizando Tecnologia de Realidade Aumentada. Revista Brasileira de Educação Especial. v. 23, n. 2, p. 215-232. 2017. Disponível em: < http://dx.doi.org/10.1590/s1413-65382317000200005>. DOI: $10.1590 / \mathrm{s} 1413-$ 65382317000200005

CAVALCANTE, L. D. W.; OLIVEIRA, G. O. B.; ALMEIDA, P. C.; REBOUÇAS, C. B. A.; PAGLIUCA, L. M. F. Tecnologia assistiva para mulheres com deficiência visual acerca do preservativo feminino: estudo de validação. Revista da Escola de Enfermagem da USP. v. 49, n. 1, p. 14-21. 2015. DOI: 10.1590/S00806234201500010002

CRUZ, D. M. C; EMMEL, M. L. G. Associação entre papéis ocupacionais, independência, tecnologia assistiva e poder aquisitivo em sujeitos com deficiência física. Revista Latino-Americano de Enfermagem, Ribeirão Preto, v. 21, n. 6, p. 484-491, abr. 2013. Disponível em: <http://dx.doi.org/10.1590/S010411692013000200003>. doi: 10.1590/S0104-11692013000200003

GAMA, G. L.; CELESTINO, M. L.; BARELA, J. A.; FORRESTER, L.; WHITALL, J.; BARELA, A. M. Effects of Gait Training with Body Weight Support on a Treadmill Versus Overground in Individuals with Stroke. Archives of Physical Medicine and $\begin{array}{llllll}\text { Rehabilitation. } & \text { n. } 98, \quad \text { p. } & 738-745 . & 2016 . & \text { DOI: }\end{array}$ http://dx.doi.org/10.1016/j.apmr.2016.11.022

GARCIA, J. C. D.; FILHO, T. A G. Pesquisa Nacional de Tecnologia Assistiva. Instituto de Tecnologia Social. São Paulo, SP: [s.n], 2012. Disponível em: $<$ http://www.santoandre.sp.gov.br/pesquisa/ebooks/368505.PDF>. Acesso em: 20 set. 2017. ISBN: 978-85-64537-04-0

GUIMARÃES, K. S. F. M.; FERREIRA, D. C. A.; SILVA, R. A. B.; SERRANO, K. V. D.; QUEIROZ, A. M.; MANTOVANI, C. P. T.; ROSSI, A. Development of an intraoral device for social inclusion of a physically disabled patient. Special Care Dentist. n. 36, v. 1, p. 53-56. 2016. Disponível em: <http://onlinelibrary. wiley.com/doi/10.1111/scd.12145/abstract;jsessionid=BE2174CD C24A9EB0349B16F62DA20AED.f04t02>. DOI: 10.1111/scd.12145 
HOHMANN, P.; CASSAPIAN, M. R. Adaptações de baixo custo: uma revisão de literatura da utilização por terapeutas ocupacionais brasileiros. Revista de Terapia Ocupacional da Universidade de São Paulo. V. 22, n.1, p. 10-18. 2011.Disponível em: <http://dx.doi.org/10.11606/issn.2238-6149.v22i1p10-18>. DOI: 10.11606/issn.2238-6149.v22i1p10-18

IBGE. Censo demográfico 2010: características gerais da população, religião e pessoas com deficiência. Ministério do Planejamento, Orçamento e Gestão. Rio de Janeiro: Instituto Brasileiro de Geografia e Estatística, 2012. Disponível em: $<$ https://biblioteca.ibge.gov.br/visualizacao/periodicos/94/cd_2010_religiao_deficienci a.pdf>. Acesso em: 29 set. 2017.

JACOB, R. T. S.; LOPES, N. B. F.; CRUZ, A. D.; ALVES, T. K. M.; SANTOS, L. G.; ANGELO, T. C. S.; CAPOANI, M. F.; MONDELLI, G.; MORET, A. L. M. TELEGRAM: contribuição na indicação de tecnologia assistiva para indivíduos com deficiência auditiva. CoDAS. v. 29, n. 1, p. 1-6. 2017. Disponível em: <http://dx.doi.org/10.1590/2317-1782/20172016012>. DOI: 10.1590/2317$1782 / 20172016012$

JACOB, R. T. S.; MOLINA, S. V.; AMORIM, R. B.; BEVILACQUA, M. C.; LAURIS, J. R. P.; MORET, A. L. M. FM listening evaluation for children: adaptação para a língua portuguesa. Revista Brasileira de Educação Especial. v. 16, n. 3, p. 359-374. 2010. Disponível em: <http://dx.doi.org/10.1590/S1413-65382010000300004>. DOI: 10.1590/S1413-65382010000300004

KAMMERS, A. C. E.; ZANETTI, A. L.; LACERDA, T. A. P.; AROCA, J. P.; CAMILOTTI, V.; MENDONÇA, M. J. Toothbrush Handles Individually Adapted for Use by Elderly Patients to Reduce Biofilm on Complete Dentures: A Pilot Study. Journal of Clinical and Diagnostic Research. v. 9, n. 5, p. 94-97. 2015. Disponível em: <https://www.ncbi.nlm.nih.gov/pubmed/26155573>. DOI: 10.7860/JCDR/2015/11261.5975

MAIA, A. M. C.; FREITAS, M. N. C. O trabalhador com deficiência na organização: um estudo sobre o treinamento e desenvolvimento e a adequação das condições de trabalho. REAd. Ed. 82, n. 3, p. 689-718. 2015. Disponível em: <http://dx.doi.org/10.1590/1413-2311.0722014.54834>. DOI: 10.1590/14132311.0722014 .54834

MAIA, F. B.; TEIXEIRA, E. R.; SILVA, G. V.; GOMES, M. K. The Use of Assistive Technology to Promote Care of the Self and Social Inclusion in Patients with Sequels of Leprosy. PLOS Neglected Tropical Diseases. 2016. Disponível em: <https://doi.org/10.1371/journal.pntd.0004644>. DOI: 10.1371/journal.pntd.0004644

MARASINGHE, K. M.; LAPITAN, J. M.; ROSS, A. Assistive Technologies for ageing populations in six low-income and middle-income countries: a systematic review. BMJ innov. n. 1, p. 182-195. 2015. Disponível em: <http://dx.doi.org/10.1136/bmjinnov-2015-000065>. DOI: 10.1136/bmjinnov-2015000065 
MEDOLA, F. O.; FORTULAN, C. A.; PURQUERIO, B. M.; ELUI, V. M. C. A new design for an old concept of weelchair pushrim. Disability and Rehabilitation: Assistive Technology. p. 1-8. 2011. Disponível em: <http://dx.doi.org/10.3109/17483107.2011.629327>. DOI: $10.3109 / 17483107.2011 .629327$

MUNIZ, L. S.; AMARAL, I. G. S.; DIAS, T. S.; JÚNIOR, J. L. R. The influence of assistive technology on occupational performance and satisfaction of leprosy patients with grade 2 disabilities. Revista da Sociedade Brasileira de Medicina Tropical. v. 49, n. 5, p. 644-647. 2016. Disponível em: <http://dx.doi.org/10.1590/0037-86820097-2016>. DOI: 10.1590/0037-8682-0097-2016

OLIVEIRA, P. M. P.; REBOUÇAS, C. B. A.; PAGLIUCA, L. ,. F. Construção de uma tecnologia assistiva para validação entre cegos: enfoque na amamentação. Revista Brasileira de Enfermagem REBEn. v. 62, n. 6, p. 837-543. 2009. Disponível em: <http://dx.doi.org/10.1590/S0034-71672009000600006>. DOI: $10.1590 / S 0034-$ 71672009000600006

PEREZ, E.; LÓPEZ, N.; OROSCO, E.; SORIA, C.; MUT, V.; BASTOS, T. F. Robust Human Machine Interface Based on Head Movements Applied to Assistive Robotics. The Scientific World Journal. p. 1-11. Disponível em: <http://dx.doi.org/10.1155/2013/589636>. DOI: 10.1155/2013/589636

RODRIGUES, P. R.; ALVES, L. R. G. Tecnologia assistiva: Uma revisão do tema. Holos, [s.l], v. 6, p.170-180, jan. 2014. Disponível em: <http://dx.doi.org/10.15628/holos.2013.1595>. doi:_10.15628/holos.2013.1595

SANTOS, S.; PEQUENO, A. A. S.; GALVÃO, C. R. C.; PESSOA, A. L. S.; ALMEIDA, E. S.; PEREIRA, J. C.; MEDEIROS, J. L. A.; KOK, F. As causas da deficiência física em municípios do nordeste brasileiro e estimativa de custos de serviços especializados. Ciência \& Saúde Coletiva. v. 19, n. 2, p. 559-568. 2014. Disponível em: <http://dx.doi.org/10.1590/1413-81232014192.00182013>. DOI: 10.1590/141381232014192.00182013

SOUZA, J. M.; CARNEIRO, R. Universalismo e focalização na política de atenção à pessoa com deficiência. Saúde e Sociedade, [s.I.], v. 16, n. 3, p.69-84, dez. 2007. FapUNIFESP (SciELO). Disponível em: <http://dx.doi.org/10.1590/S010412902007000300007>. doi: 10.1590/S0104-12902007000300007

VARELA, R. C. B.; OLIVER, F. C. A utilização de Tecnologia Assistiva na vida cotidiana de crianças com deficiência. Ciência \& Saúde Coletiva, Rio de Janeiro, v. 18,n. 6, p.1773-1784, jun. 2013. Disponível em: <http://dx.doi.org/10.1590/S141381232013000600028>.doi: 10.1590/S1413-81232013000600028 